EPJ Web of Conferences 74,03001 (2014)

DOI: 10.1051/epjconf/ 20147403001

(C) Owned by the authors, published by EDP Sciences, 2014

\title{
Light and/or atomic beams to detect ultraweak gravitational ef- fects
}

\author{
Angelo Tartaglia ${ }^{1,2, a}$, Jacopo Belfi ${ }^{3}$, Nicolò Beverini ${ }^{3,4}$, Angela Di Virgilio ${ }^{3}$, Antonello Ortolan ${ }^{5}$, \\ Alberto Porzio ${ }^{6}$, and Matteo Luca Ruggiero ${ }^{1,2}$ \\ ${ }^{1}$ Department of Applied Science and Technology, Politecnico di Torino, Italy \\ ${ }^{2}$ INFN, Sezione di Torino, Italy \\ ${ }^{3}$ INFN, Sezione di Pisa, Pisa, Italy \\ ${ }^{4}$ Department of Physics, University of Pisa, Pisa, Italy \\ ${ }^{5}$ INFN, Legnaro National Laboratory, Italy \\ ${ }^{6}$ CNR-SPIN, Naples, Italy and INFN, sezione di Napoli, Italy
}

\begin{abstract}
We shall review the opportunities lent by ring lasers and atomic beams interferometry in order to reveal gravitomagnetic effects on Earth. Both techniques are based on the asymmetric propagation of waves in the gravitational field of a rotating mass; actually the times of flight for co- or counter-rotating closed paths turn out to be different. After discussing properties and limitations of the two approaches we shall describe the proposed GINGER experiment which is being developed for the Gran Sasso National Laboratories in Italy. The experimental apparatus will consist of a three-dimensional array of square rings, $6 m \times 6 m$, that is planned to reach a sensitivity in the order of $1 \mathrm{prad} / \sqrt{ } \mathrm{Hertz}$ or better. This sensitivity would be one order of magnitude better than the best existing ring, which is the G-ring in Wettzell, Bavaria, and would allow for the terrestrial detection of the Lense-Thirring effect and possibly of deviations from General Relativity. The possibility of using either the ring laser approach or atomic interferometry in a space mission will also be considered. The technology problems are under experimental study using both the German G-ring and the smaller G-Pisa ring, located at the Gran Sasso.
\end{abstract}

\section{Introduction}

General Relativity (GR) is a fascinating intellectual framework describing the material world at the widest scale. It is a classical theory whose revolutionary feature is the new way of interpreting time (already implemented in Special Relativity) and the 'geometrization' of the universal gravitational interaction. Confronted with its internal consistency and elegance and its worldwide fame the direct verifications of genuine GR effects are comparatively few, at least when confronted with other theories. This is probably due to the fact that the effects directly observable on or from earth dwell in general in the domain of weak or ultra-weak energies.

Historically the first success of GR was obtained in 1915 (and published in 1916) [1] explaining the anomalous precession of the perihelion of Mercury, known to astronomers since a long time but

\footnotetext{
a e-mail: angelo.tartaglia@polito.it
} 
lacking a consistent explanation. The already measured precession rate, exceeding the contributions due to the interaction with the other bodies of the solar system and the quadrupole of the sun, was 43 "/Julian century and the prediction of GR was indeed 43"/Julian century. The next and most resounding hit of GR was, in 1919 [2], the verification of the predicted lensing effect by the sun. In 1915 Einstein had noticed that, according to his GR theory, the deviation of the light rays grazing the sun would have been twice as big as the prediction of Newtonian gravity, and that was indeed the case.

Another interesting effect predicted by GR is the reddening of light coming from regions where a gravitational field is present. This effect was first recognized in 1925 observing the spectral lines of the light coming from Sirius B [3], then, more than 30 years later, it was directly measured on earth by Pound and Rebka [4].

The often called 'fourth classical test' of GR is the delay in the time of flight of electromagnetic signals traveling across a gravitational field. This effect, also known as Shapiro time delay, was measured for the first time in 1971 [5].

Out of the solar system an interesting test bed for GR are binary systems where gravity is strong, as it happens when at least one of the member stars is a compact object. Strong evidence for relativistic precession of the periapsis has been obtained from the observation of the PSR B1913+16, the first binary pulsar discovered in 1974: the entity of the precession is as big as 4.2 degrees/year. An even stronger evidence comes from PSR J0737-3029, the only so far discovered double pulsar; here the precession of the periapsis is 16.9 degrees/year. It must be mentioned that this data is used to obtain information about other features of the pair of stars, first of all the masses; there is then some circularity between the value one deduces for the masses and the evaluation of the masses themselves. In short it would be more correct to say that the measured precession is (strongly) compatible with GR. The same attitude may be adopted, in the case of the double pulsar, for the geodetic (or de Sitter) precession (the precession induced by the interaction of the angular momentum of a gyroscope and the gravitational field in which it moves): there too the observations are compatible with GR.

In any case the most spectacular indirect confirmation of GR comes from the observation of the binaries with pulsars. One of the predictions of GR is the existence of gravitational waves (GW) and many experiments have been made or are under way, aiming to the detection of the waves. As it is well known, for the moment no positive result has been obtained because of the extreme weakness of the expected signals, however if we look at the time decay of the orbital periods of pulsars in binaries we find that the observed curves correspond astonishingly well to what expected from a quadrupole emission of GW from the pair of stars. Fig. 1 clearly shows the correspondence between data and theory [6] ${ }^{1}$. The agreement, in the case of PSR B1913+16, is within $0.2 \%$.

Another class of weak effects of GR includes phenomena due to the motion of the source of gravity and in particular, excluding observer dependent effects, due to the rotation of the source. These effects may be grouped under the common heading of gravito-magnetic phenomena, since, when working in the weak energy domain and linearizing Einstein's equations of gravity, the latter come to look similar to Maxwell's equations and the interaction we are speaking of is the analog of the magnetic one. In the rest of this talk we will concentrate on gravito-magnetism and the ways to reveal it. In particular we will illustrate the possibility given by ring-lasers, describing the GINGER experiment that is now under implementation in Italy. As we shall see the principle exploited by ring-lasers can also work for waves other the electromagnetic ones and in particular with atomic beams, which could hint to a promising technique for the future.

\footnotetext{
${ }^{1}$ The curve for PSR J0737-3029 is due to the courtesy of A. Possenti
} 

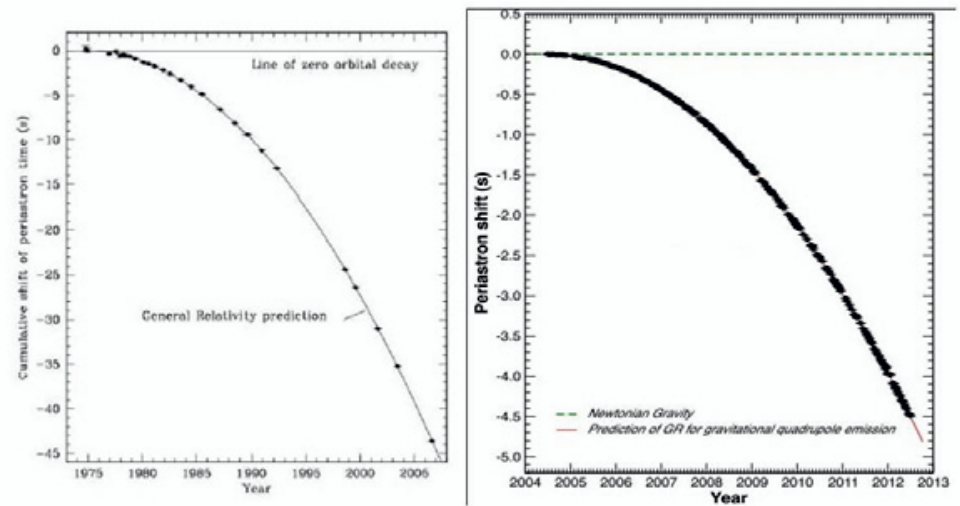

Figure 1. Orbital period decay of PSR B1913+16 (left) and PSR J0737-3029 (right).

\section{Rotating sources of gravity}

The properties of the space-time surrounding a steadily rotating mass are expressed by the general axisymmetric line element:

$$
d s^{2}=g_{t t} d t^{2}+g_{r r} d r^{2}+g_{\theta \theta} d \theta^{2}+g_{\phi \phi} d \phi^{2}+2 g_{t \phi} d t d \phi
$$

Space polar coordinates have been used in order to manifest the symmetry. All $g_{\mu \nu}$ 's depend on $r$ and $\theta$ only. As for gravito-magnetic effects the relevant term is $g_{t \phi}$. For a real massive source no global coordinate transformation exists reducing (1) to the Minkowski line element. If we use other space coordinates $\{x\}$, the general form of the off-diagonal elements of the line element will be $2 g_{t i} d t d x^{i}$ and of course the index $i$ runs from 1 to 3 . In the three space dimensions the latter expression may be interpreted as the scalar product of a three-vector $\vec{h}$ times the space tangent vector $\overrightarrow{d x}$. Keeping the analogy with electromagnetism $\vec{h}$ is treated as the gravito-magnetic (three-)vector potential.

In the solar system the gravitational field, and the more the gravito-magnetic term, is very week so that it is possible to use an approximated version of the line element. Supposing that the deviations from the spherical symmetry in the mass distribution can be negligible, the approximated line element can be written:

$$
\begin{aligned}
d s^{2} \simeq\left(1-2 G \frac{M}{c^{2} r}\right) c^{2} d t^{2}-\left(1+2 G \frac{M}{c^{2} r}\right) d r^{2}-r^{2} d \theta^{2}-r^{2} \sin ^{2} \theta d \phi^{2} & \\
& +4 G \frac{J}{c^{3} r^{2}} \sin \theta(c d t)(r \sin \theta d \phi)
\end{aligned}
$$

In the formula we have evidenced the elements of the physical space basis forms $(d r, r d \theta, r \sin \theta d \phi) . G$ is Newton's constant, $M$ is the central mass and $J$ is the angular momentum 
of the source. The gravito-magnetic vector potential has the only component $h_{\phi}=2 G \frac{J}{c^{3} r^{2}} \sin \theta$. Let us evaluate the numerical values of the relevant parameters, in shorthand notation:

$$
\mu=G \frac{M}{c^{2}} \approx 4.43 \times 10^{-3} \mathrm{~m} ; \quad j=G \frac{J}{c^{3}} \approx 1.75 \times 10^{-2} \mathrm{~m}^{2}
$$

On the surface of the earth (radius $R$ ) the deviation from flat space-time is measured by $\mu / R \sim 10^{-9}$ and $j / R^{2} \sim 10^{-16}$. One must be very careful in considering the approximation orders especially in the first two terms on the right of eq. (2) in order not to neglect contributions which are the same order or bigger than the off-diagonal term.

\subsection{Past and present tests of gravito-magnetic effects}

Rotation effects in relativity include the Sagnac effect, which is a purely kinematical effect due to the motion of light along a closed circuit whenever the components of the latter are rotating (or in motion with respect to the source of light). Properly GR effects are present whenever the gravitational field is non-negligible and the off-diagonal time-space elements of the metric tensor are non-zero. There are two such effects: the geodetic precession (or de Sitter precession) which is due to the coupling between the motion of a gyroscope and the gravito-electric (i.e. Newtonian) component of the gravitational field; the Lense-Thirring (LT) or frame-dragging effect, induced by the off-diagonal time-space terms of the metric tensor, i.e. by the gravito-magnetic component of the gravitational field.

The detection of the Sagnac effect goes back to Sagnac's experiments in 1913 [? ]. The direct measurement of the GR effects is much more recent. The LT drag originating from the rotation of the earth has been evaluated analyzing the orbital behavior of the two LAGEOS satellites, with an accuracy of $10 \%$. A dedicated experiment has been done by the space mission Gravity Probe B (GPB) which verified the de Sitter precession with the accuracy of $0.2 \%$ and the LT drag at the level of 19\%. The dedicated LARES space mission is now under way and taking data with the aim of testing LT with the accuracy of $1 \%$. Other evidence for the frame dragging effect, though with lower and difficult to assess accuracy, comes from the lunar laser ranging; furthermore results compatible and consistent with LT, are obtained from the study of the orbital dynamics of pulsars in binaries and in particular from the double pulsar.

So far, as we have seen, the number of direct tests is quite low and the accuracy not very high, so that new experiments with different techniques and strategies are welcome. The approach we shall describe in the next sections is based on the use of ring-lasers as extremely sensible rotation sensors.

\section{Propagation of light in a rotating system}

Suppose you have a light beam which is led, by properly located mirrors, to move along a path closed in space. We may ask how long does it take to complete one turn along the contour. The answer is found starting from eq. (1), equating it to zero (since the worldlines of electromagnetic signals are null) and solving for the coordinated time differential $d t$. The next step is to integrate along the flight path of the beam. It is immediately clear that there are two different results depending on the rotation sense of light with respect to the orientation of the rotation vector of the gravity source. The time of flight difference between the co-rotating and the counter-rotating beams is:

$$
\delta t=-\frac{2}{c} \oint \frac{g_{t i}}{g_{t t}} d x^{i}
$$

The relevant quantity in a laboratory should be expressed in terms of the proper time of an observer in the lab, rather than in coordinated time; so the practical formula will be: 


$$
\delta \tau=-\frac{2}{c} \sqrt{g_{t t}} \oint \frac{g_{t i}}{g_{t t}} d x^{i}
$$

A tool for evidencing the time difference (5) could be an interferometer, as it was the case for the former Sagnac effect, but now it is possible to work with ring lasers. The scheme of a (square) ring laser is visible in fig. 2.

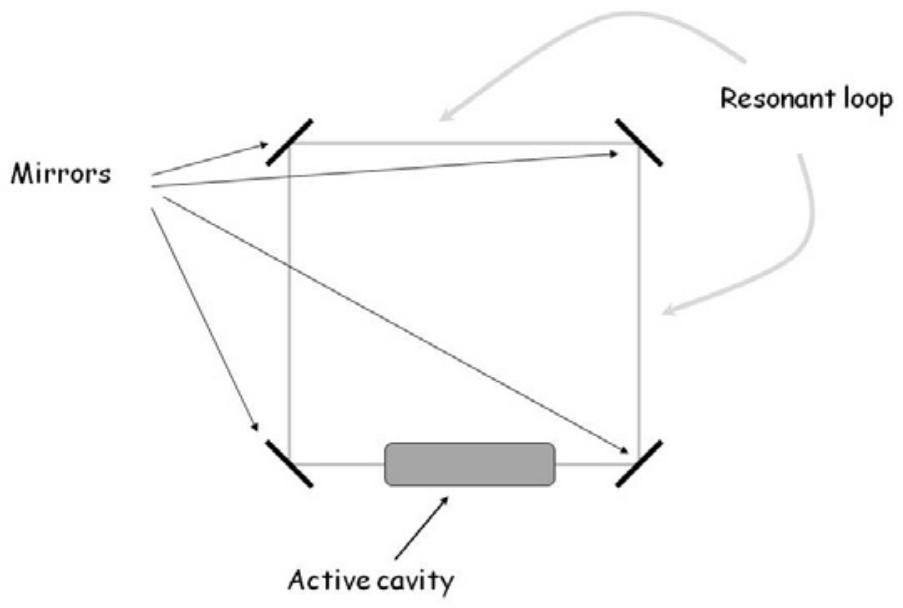

Figure 2. Scheme of a square ring laser

In the ring one has a stationary superposition of right- and left-handed coherent standing waves. However now the time of flight difference, in each standing wave, appears as a frequency difference and the difference gives in turn rise to a beat frequency that can be extracted and read at one of the corners of the ring. In terms of the beat frequency $v_{b}$ one has:

$$
v_{b}=\frac{c^{2}}{\lambda P} \delta \tau
$$

The additional parameters which appear in the formula are the fiducial wavelength of the laser $\lambda$ and the length of the path traveled by light $P$. Converting the line element to local coordinates of a laboratory on earth (which of course is non-inertial) and introducing the approximations inherent to the smallness of the relevant gravitational terms down to the corrections including the angular momentum of the planet, the expected beat frequency readable in a ring laser will be[8]:

$$
v_{b}=2 \frac{A}{\lambda P} \vec{\Omega} \cdot \hat{u}_{n}-4 \frac{A}{\lambda P} \frac{\mu}{R} \Omega \sin \theta \hat{u}_{\theta} \cdot \hat{u}_{n}+2 c \frac{A}{\lambda P} \frac{j}{R^{3}}\left(2 \cos \theta \hat{u}_{r}+\sin \theta \hat{u}_{\theta}\right) \cdot \hat{u}_{n}
$$

The quantity $\frac{A}{\lambda P}$ is called 'scale factor': the bigger it is the higher is the response of the ring. $\vec{\Omega}$ is the rotation vector of the earth; the angle $\theta$ is the colatitude of the location of the ring laser; the $\hat{u}$ 's are unit vectors along, respectively, the perpendicular to the plane of the ring $(n)$, the radial direction from the center of the earth $\left(_{r}\right)$, the direction of the local meridian $\left(_{\theta}\right)$. 
The scale factor, in all terms of eq. (7), multiplies quantities having the dimensions of angular velocities. The first term is the classical Sagnac contribution; the other two terms are the physical GR ones and the last is the one properly gravito-magnetic (LT term). For a side of the square in the order of $4 \mathrm{~m}$ or more, the beat frequency is of the order of a hundred $\mathrm{Hz}$ for Sagnac and $10^{-9}$ smaller for the other two contributions. In practice, in order to reveal the GR terms a sensitivity in the order of one $\mathrm{pHz}$ is needed.

\section{Existing ring lasers}

Ring lasers already exist in various forms, from small commercial ones to big research devices. In all cases they are used as sensors of rotations. The commercial ones have replaced old mechanical gyroscopes (hence the name of gyrolasers) on board of airplanes or even ships and serve as controllers of trim, pitch and roll angles. Their sensitivity is $\sim 10^{-7} \mathrm{rad} / \mathrm{s} \times \sqrt{\mathrm{Hz}}$.

The bigger and more sensitive rings are used for geophysics and geodesy, as sensors of the small local rotations of the crust of the earth; they are of course also able to measure the diurnal angular velocity of the planet. The best existing ring at the moment is the gross Ring G located in Wettzell, Bavaria. $\mathrm{G}$ is a square, $4 \mathrm{~m}$ side, mounted on a monolithic zerodur (an extremely rigid and thermally stable ceramic material) slab, and located in a laboratory under an artificial mound in order to screen the device from superficial rotational noise. The laser power is $20 \mathrm{nW}$; $\mathrm{G}$ has already achieved a sensitivity of a few $\mathrm{pHz}$ and is able to reveal the diurnal polar motion of the axis of the earth[9].

It is worth mentioning also the rings build by the group of the University of Canterbury in Christchurch (NZ) in the Cashmere Cavern [10]. They arrived to $20 \mathrm{~m}$ long-side rectangles $\left(367.5 \mathrm{~m}^{2}\right.$ area), thus obtaining the biggest scale factor; unfortunately various instabilities (starting from the mechanical ones) overwhelm the advantage of a huge scale factor.

Another ring which is now used both for testing technologies and for characterizing the site where the new GINGER instrument (described in the next section) will be placed, is G-Pisa [11]. It is a square, $1.35 \mathrm{~m}$ side, mounted on a granite slab, that was initially build for characterizing, from the viewpoint of rotational noise, the site of the Gravitational Waves VIRGO interferometer in Cascina near Pisa (Italy). G-Pisa, that has now been moved to the Laboratori Nazionali del Gran Sasso (LNGS) in Italy, has a sensitivity of a few hundreds $\mathrm{prad} / \mathrm{s} \times \sqrt{\mathrm{Hz}}$.

\section{GINGER}

Considering the achievements of $\mathrm{G}$ and the tremendous progresses obtained in recent years in laser technologies the aim of measuring the LT drag and GR rotational effects in general appears to be attainable. This is the reason why a collaboration including the institutions of the authors of the present paper (p.i. Angela Di Virgilio ) together with the G group at the Technische Universität München (p.i. Ulrich Schreiber) and the University of Canterbury (NZ) group (p.i. Jon Paul Wells) has started to implement a new experiment, named GINGER (Gyroscopes IN GEneral Relativity). GINGER will be dedicated primarily to fundamental physics for testing GR and possibly deviations from GR [8]. The physical terms appearing in eq. (7) are indeed vectors with different orientations in space, so GINGER will not be a single high sensitivity ring, but a three-dimensional array of square rings in order to be able to measure all space components of the relevant vectors. Two possible configurations are shown in fig. 3.

The side of each ring will be $6 \mathrm{~m}$ in order to increase the scale factor. Because of the size and of the three-dimensionality it is impossible to think of a sufficiently rigid and stable monolithic structure, so a heterolithic (i.e. with mirrors mounted on in principle independent supports) configuration is devised. 


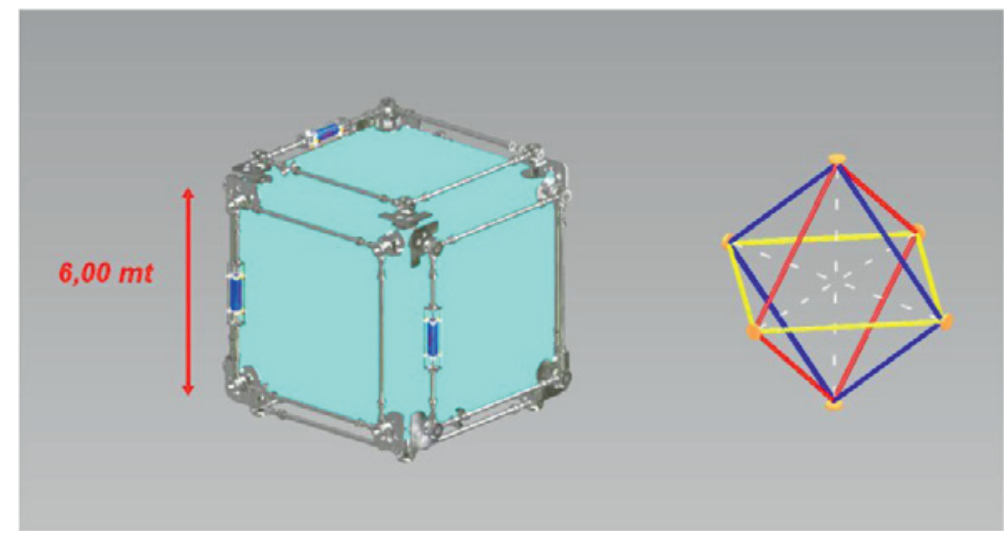

Figure 3. Possible three-dimensional configurations for GINGER. On the left a cube with a ring on each face (a factor of 2 redundancy); on the right an octahedron with geometry control along the three diagonals.

The shape and geometry control will be obtained dynamically by means of piezoelectric actuators on each mirror; in the case of the octahedron it is more easily understandable the way by which the control will be performed; each diagonal of the octahedron will be a Fabry-Pérot cavity and the signal from the cavity will be used as a feed back in order to pilot the piezos of the mirrors at the end of the cavity, keeping the length stable. The dynamical control is already under test on G-Pisa. The power of the lasers will not exceed $200 \mathrm{nW}$ with a cavity quality factor $Q=3 \times 10^{12}$. The location of GINGER will be at the LNGS, under $1400 \mathrm{~m}$ of rock, thus drastically reducing the external noise. As we have written above G-Pisa has now been moved to the LNGS in order to study in situ the response of the ring to the environment of the laboratory and to test the dynamical control of the geometry. The next step will be (within 2014) the building of a GINGER pathfinder (GINGERino) consisting in a single $3.6 \mathrm{~m}$ side ring with the purpose of studying the stability of the laser, the behaviour of the mirrors, etc.

Finally between 2015 and 2016 GINGER will be built and will start working and collecting data.

\section{Other experimental approaches to the General Relativistic effects on earth}

\subsection{Matter waves interferometry}

The anisotropic propagation of waves in the gravitational field of a rotating body is not limited to light. The time of flight difference between right- and left-handed closed paths is in principle there for any kind of wave; in particular it affects also the propagation of quantum atomic beams. There is only a slight difference in the formulae due to the fact that the propagation speed is $<c$ so that the line element is timelike. In practice the most appropriate technique one could use is interferential: an appropriate crystal lattice splits an atomic beam into two sub-beams in a horizontal plane; the diffraction onto another lattice leads the two sub-beams to superpose again. If the apparatus is rotating or is immersed in the gravitational field of a rotating body, even though the paths followed by the two half beams are geometrically equal the final phases will be different and an interference pattern will be produced. The phase difference is proportional to the kinematical or effective (or both) rotation rates. As in the case of light the phase difference is proportional to the area delimited by the two beams and explicitly is: 


$$
\delta \phi=\frac{4 \pi}{h} m A \vec{\Omega}_{\text {effective }} \cdot \hat{u}_{n}=\frac{4 \pi}{c \lambda} \vec{\Omega}_{\text {effective }} \cdot \hat{u}_{n}
$$

In the formula, $m$ is the mass of the particles of the beam and $h$ is Planck's constant; $\lambda=m c^{2} / h$ is the wavelength of the particles; $\vec{\Omega}_{\text {effective }}$ includes all effects, both kinematical and gravitational, expressed as equivalent rotation vectors. Now the size of the device is much smaller than in the case of a ring laser but the wavelength is much much smaller than that of light, so that in the end the multiplicative factor can be bigger. The weak point is the difficulty to have stable, long lasting conditions for the beam.

The atomic beams interference is an interesting technique, which at the moment is not yet fully implemented but could become relevant soon.

\subsection{Ring lasers in space}

The experiment in a laboratory fixed to the earth uses necessarily a non-inertial reference frame; furthermore the interesting physical signals are extremely small constant (DC) perturbations on top of the dominant kinematical Sagnac effect directly measuring the rotation rate of the earth. In practice better experimental conditions could be obtained on a freely falling (i.e. orbiting) laboratory. A possibility could be to have a compact tetrahedral configuration made of four triangular ring lasers carried by a satellite orbiting the earth. This solution would allow to modify the spin of the spacecraft thus controlling, modulating or even suppressing the Sagnac term, and facilitating the emergence of the GR signals.

\subsection{Linear cavities}

In principle not only a ring described in space by light rays is able to reveal the gravito-magnetic part of the gravitational field. Considering the situation in four dimensions, light bouncing back and forth in a linear cavity identifies a rhomboidal space-time patch delimited by the null world-lines of the rays. The situation is better understood looking at fig. 4 .

The changes in the electromagnetic tensor of the waves, induced by gravity, are given by the contraction of the Riemann tensor with the space-time area $S$ (an antisymmetric rank 2 tensor) delimited by the light beam. In formulae it is:

$$
\delta F^{\mu \nu}=\left(R_{\epsilon t i}^{\mu} F^{\epsilon v}+R_{\epsilon t i}^{v} F^{\mu \epsilon}\right) \delta S^{t i}
$$

$R_{\alpha \beta v}^{\mu}$ are the elements of the Riemann tensor; $F^{\alpha \beta}$ is the electromagnetic tensor; Greek indices run from 0 to 3 , the $i$ index runs from 1 to 3 .

As an example let us consider a cavity aligned along the local meridian and let us start with a linearly polarized wave with an East-West magnetic field component. Let us then express the Riemann tensor in the local reference frame using the approximation introduced in eq. (2). We get:

$$
\delta F^{\theta \phi} \simeq\left[\left(\frac{\mu \Omega}{c R^{2}}-3 \frac{j}{R^{4}}\right) \frac{\cos \theta}{\sin ^{2} \theta}\right] \frac{l^{2}}{R} F^{\theta r}
$$

The square bracket contains the linearized version of $R_{r t \theta}^{\phi} ; l$ is the length of the cavity. We see that gravity induces a radial (with respect to the center of the earth) magnetic field in the wave, cumulating at each bounce on the mirrors. The effects appear as polarization changes in the waves. Estimating the fractional changes in $F$ at the surface of the earth each time light goes through the cavity we obtain, 


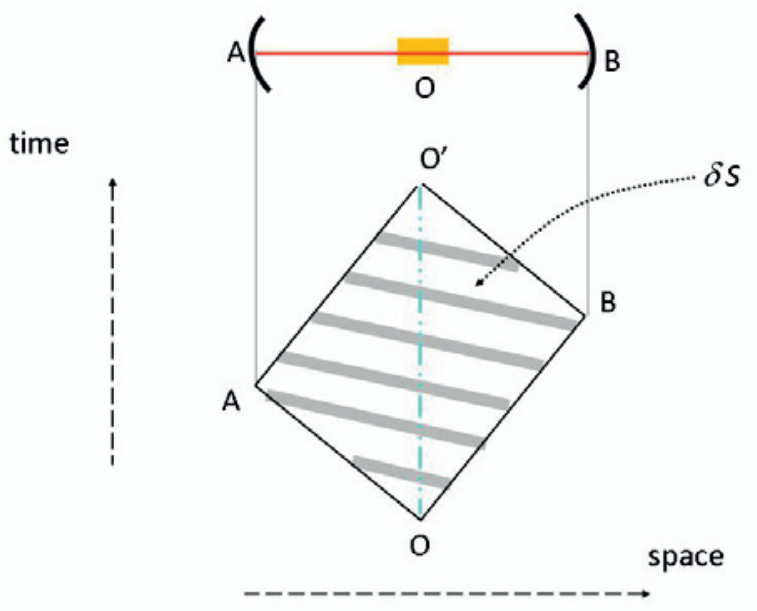

Figure 4. A linear resonating cavity with a light beam between two mirrors is schematically drawn in the upper part of the figure. Below the same situation is described in space-time. The world-lines of the light rays identify a space-time area.

as a kinematical effect of the rotation of the laboratory with respect to the fixed stars, $\delta F / F \sim 10^{-7}$; as an effect of the gravito-electric (i.e. Newtonian) field, $\delta F / F \sim 10^{-8}$; finally, because of the gravitomagnetic component of the field, $\delta F / F \sim 10^{-14}$.

Even though at the moment it is not clear how the information could practically be read out of the cavity, the effects are there and it is worth to continue the investigation in view of an actual experimental implementation of the idea.

\subsection{An orbital gyro}

Here we present one more possibility offered by the abundance of artificial satellites orbiting the earth. The European Galileo positioning system, whose fleet is under deployment, is, for instance, proposing itself as open also to fundamental physics experiments. Suppose then to have three or more satellites on approximately the same orbital plane, equipped with transponders enabling the reception and re-sending of electromagnetic signals from one to another. In fact the system can work as a gigantic electromagnetic gyroscope, provided a light (or radio) signal starting from one spacecraft is sent in two opposite senses towards the others. If you are able to measure the time of flight for the complete tour of the satellites, you will find a right/left difference. In the case of a circular equatorial orbit of radius $R_{o} \sim 10^{7} \mathrm{~m}$ the time difference due to both the de Sitter and the LT effects would be approximately one hundredth of the period of light $T$ (using laser pulses):

$$
\delta \tau \simeq-2 \frac{A}{c R_{o}}\left(\left(\frac{\mu}{R_{o}}\right)^{3 / 2}-2 \frac{j}{R_{o}^{2}}\right) \sim 10^{-2} T
$$




\section{Conclusion}

We have reviewed various possibilities to test GR effects due to the rotation of the earth, especially focalizing on one technique, the measurement of effective rotations by means of ring-lasers, and one experiment, GINGER. In general light is a very good probe for relativity being an intrinsically relativistic entity.

GINGER will be a three-dimensional array of square ring lasers, encompassing a $36 \mathrm{~m}^{2}$ area each and will be located underground at the LNGS site in Italy. The control of the geometry will be obtained dynamically using resonating cavities along the diagonals of the squares to provide a feedback signal to operate piezoelectric positioning devices for the mirrors. The aim is to measure the LT effect with an accuracy better than $1 \%$ and possibly to set new upper limits to the deviations from GR. The road to the full implementation of GINGER is already under way; the project has been proposed by groups belonging to various universities and research institutions in Italy (INFN; CNR; Universities of Florence, Naples, Padua, Pisa, Politecnico University of Turin), together with the Technische Universiät München in Germany and the University of Canterbury in New Zealand.

A strong point of GINGER with respect, for instance, to experiments in space is its comparatively low cost and the possibility to operate hands on the device, modifying settings and correcting mistakes while working. First results are expected in 2015-2016 and we are confident they will come and be interesting.

\section{References}

[1] A. Einstein, Annalen der Physik 49 (7), 769-822 (1916)

[2] F. W. Dyson, A. S. Eddington, C. Davidson, Philosophical Transactions of the Royal Society 220A, 291-333 (1920)

[3] W. S. Adams, Proc. natn. Acad. Sci. USA 11, 382 (1925)

[4] R. V. Pound, G. A. Rebka Jr, Phys. Rev. Lett. 3 (9), 439-441 (1959)

[5] I. I. Shapiro, et al., Phys. Rev. Lett. 26 (18), 1132-1135 (1971)

[6] J.M.Weisberg, J.H. Taylor, "The Relativistic Binary Pulsar B1913+16: Thirty Years of Observations and Analysis", in ASP Conference Series 328, F.A. Rasio and I.H. Stairs (eds.), 25 (2005)

[7] M. G. Sagnac, Comptes Rendus 157, 708-710 (1913);

M. G. Sagnac, Comptes Rendus 157, 1410-1413 (1913)

[8] F. Bosi, et al., Phys. Rev. D, 84, 122002-1-122002-23 (2011)

[9] K. U. Schreiber et al., Pure Appl. Geophys. 166, 1485-1498 (2009)

[10] C. H. Rowe, U. K. Schreiber, S.J.Cooper, B.T.King, M.Poulton and G.E.Stedman, Appl. Opt. 38 , 2516-2523 (1999)

[11] A. Di Virgilio, et al.,Class. Quantum Grav., 27, 4033 (2010) 